\title{
NEUROIMAGING IN NEWLY DIAGNOSED EPILEPSY
}

The use of neuroimaging and its value in diagnosis were determined in a community based study of 613 children with newly diagnosed epilepsy referred to Yale University, New Haven, CT. Of the patients examined, $80 \%$ had imaging studies, $63 \%$ received an MRI, $32 \%$ a CT scan, and $16 \%$ had both. Idiopathic generalized epilepsies and other forms of epilepsy were evaluated with imaging studies in $50 \%$ and $87 \%$ of cases, respectively. Neuroimaging was positive for etiologically relevant abnormalities in $12.7 \%$ of cases. Of 14 patients whose clinical findings were otherwise completely normal, neuroimaging showed tuberous sclerosis in 4, tumors (2), arteriovenous abnormality (1), cavernous angioma (1), cerebral malformations (3), and other lesions (3). Seizures were partial in 13 (93\%) of the 14 cases, and EEGs were focal in 12 (96\%). (Berg AT, Testa FM, Levy SR, Shinnar S. Neuroimaging in children with newly diagnosed epilepsy: a community-based study. Pediatrics September 2000;106:527-532). (Reprints: Anne T. Berg PhD, Department of Biological Sciences, Northern Illinois University, DeKalb, IL 60115).

COMMENT. Etiologically relevant cerebral abnormalities may be detected by imaging studies in a small but significant proportion of newly diagnosed epilepsies in children, despite an otherwise normal clinical presentation. Patients with neurologic deficits, partial seizures, or focal EEG abnormalities are especially at risk for positive imaging studies.

\section{ELECTRICAL STATUS EPILEPTICUS DURING SLEEP}

Eighteen children with medically refractory seizures, language deterioration, incoordination, and regression in behavior and intellect, and an EEG showing electrical status epilepticus during sleep (ESES), are reported from Beijing Medical University, and the University of Hong Kong, Republic of China. Age at seizure onset ranged from 7 months to 9 years, and ESES was diagnosed at $8.5+/-4.3$ years (range $=3-21$ yrs) after onset of seizures. Development of ESES signaled the appearance of neuropsychological, language, and behavioral deterioration. Underlying epileptic syndromes included Lennox-Gastaut (4), Landau-Kleffner (3), benign rolandic epilepsy variant (5), and epileptiform autistic regression in 3,1 having Rett syndrome. Treatment with oral or intravenous clonazepam resulted in improvement in clinical and EEG manifestations, especially when treated within 2 years of seizure onset. Valproic acid was also effective in one half the patients, while carbamazepine, phenytoin, and phenobarbital were without benefit. Improvements in speech and cognitive function were correlated with suppression of ESES. (Liu XY, Wong V. Spectrum of epileptic syndromes with electrical status epilepticus during sleep in children. Pediatr Neurol May 2000;22:371-379). (Respond: Professor Virginia Wong MD, Division of Neurodevelopmental Paediatrics, Queen Mary Hospital, University of Hong Kong, Hong Kong, Peoples Republic of China).

COMMENT. The EEG phenomenon of electrical status epilepticus during sleep (ESES) in childhood may be associated with epileptic syndromes of various etiologies and outcomes. Benign rolandic epilepsy and Lennox-Gastaut syndromes represent the benign and malignant ends of the spectrum. The authors emphasize the importance of sleep EEG monitoring in children with an early onset of epilepsy associated with language, behavioral or psychological deterioration. Early treatment with oral benzodiazepines, especially clonazepam or clobazam, 
may reverse both the EEG and cognitive, language, and behavioral regression. The well known tendency to development of tolerance with use of benzodiazepines was considered unusual in the treatment of ESES in this study. Seizures and the ESES may be exacerbated by carbamazepine.

\section{VALPROATE-INDUCED WEIGHT GAIN}

To determine the relation of carnitine and insulin levels to weight gain during valproate therapy of epilepsy in children, twenty patients were randomly assigned to receive either carnitine or placebo supplementation, in a study at Hacettepe University, Ankara, Turkey. The mean age was 8.3 and 9.4 years in the two groups. Seizures were completely controlled. Weight gain occurred in both groups after a 3 month period of observation. Weight gain did not correlate with carnitine levels. Mean insulin levels and insulin/glucose ratios were increased during valproate therapy. Weight gain might be related to the decrease in glucose levels and consequent appetite stimulation. (Demir E, Aysun S. Weight gain associated with valproate in childhood. Pediatr Neurol May 2000;22:361-364). (Respond: Dr Sabiha Aysun, Department of Pediatric Neurology, Hacettepe University, Ankara 06100, Turkey).

COMMENT. Weight gain associated with valproate therapy for epilepsy is not related to carnitine deficiency. It may be caused by appetite stimulation, secondary to higher insulin and lower blood glucose levels. VPA may decrease glucose levels by inhibiting gluconeogenesis, and by other possible mechanisms.

\section{TOXIC-METABOLIC DISORDERS}

\section{PRENATAL ALCOHOL AND NEUROLOGIC DYSFUNCTION}

Of 698 pregnant women interviewed about alcohol consumption at the maternity hospital in Roubaix, France, 156 of the offspring were investigated at age 4 and $1 / 2$ years, using a standardized neuropsychological assessment. A posture score was not related to alcohol consumption, whereas a lower general cognitive index $(\mathrm{GCI})$ and a minor neurological sign score were directly related to consumption of $21 \mathrm{drinks/week} \mathrm{(3} \mathrm{drinks/day).} \mathrm{The} \mathrm{minor} \mathrm{neurogic} \mathrm{signs} \mathrm{found}$ at the neuromotor evaluation included synkinesis or mirror movements, fingernose incoordination, hopping dyspraxia, and impaired ability to walk on heels. The high number of neurologic abnormalities was associated with prenatal alcohol consumption, after controlling for a lower GCI. (Larroque B, Kaminski M, Dehaene P, Subtil D, Querleu D. Prenatal alcohol exposure and signs of minor neurological dysfunction at preschool age. Dev Med Child Neurol August 2000;42:508-514). (Respond: Beatrice Larroque MD PhD, Institut National de la Sante et de la Recherche Medical Unit 149, 16 Avenue Paul Vaillant, Couturier, 94807 Villejuif Cedex, France).

COMMENT. Children born to mothers who consumed 21 or more alcoholic drinks per week ( $11 \%$ of the sample) had more minor neurologic abnormalities than those exposed to less alcohol. Levels of alcohol consumption lower than those associated with fetal alcohol syndrome can cause impairments of neurologic function recognized on clinical examination.

Twelve-year follow-up of children exposed to alcohol in utero, at the University of Helsinki, found that the longer the exposure and the more severe the fetal alcohol syndrome, the more often the children required special 